\title{
Los estudios hispánicos en algunos países de América del Sur. Tendencias actuales de la investigación y perspectivas futuras
}

\section{Melchora Romanos}

Arbor CLXVIII, 664 (Abril 2001), 533-549 pp.

\section{Introducción}

Todo intento de abarcar un panorama descriptivo de la situación actual de los estudios hispánicos en América del Sur, resulta ante todo una tarea realmente ciclópea e imposible de realizar, por cuanto se requeriría contar con una red de información y un sistema de comunicaciones fluidas entre los diversos centros de investigación y universidades que, al menos por el momento, no existen. La enorme extensión geográfica que dificulta los contactos personales e institucionales, los problemas políticos y económicos en que se encuentran inmersos los países de la región atomizan la unidad que, por razones históricas y culturales, debería configurar las señas de identidad común. Estas circunstancias insoslayables determinan la necesidad de establecer las limitaciones y las pautas con las que me propongo abordar este enfoque, necesariamente parcial y muy acotado, del objetivo propuesto en principio por el coordinador de esta publicación:

$\mathrm{Al}$ margen de lo que estas consideraciones significan como diagnóstico impracticable del hispanismo en esta región de América, creo que es sumamente sintomático observar que, por ejemplo, en el Directorio de socios de la Asociación Internacional de Hispanistas (1998), los únicos países que cuentan con miembros asociados son: Argentina (58 socios); Brasil (16 socios); Chile (3 socios); Ecuador (1 socio); Paraguay (1 socio); Perú (2 socios); Venezuela (5 socios). A esta evidente desproporción se agrega 
como un hecho significativo que en el Boletín (4/97) de la misma Asociación se cuente tan solo con información bibliográfica y de interés general sobre las actividades académicas de Argentina y Uruguay a cargo de Juan Diego Vila, de Brasil a cargo de Antonio R. Esteves y de Perú a cargo de Teodoro Hempe Martínez. Esto prueba que las relaciones de los hispanismos iberoamericanos con el hispanismo internacional se sustentan de forma más sólida en unos pocos países mientras que es evidente que existe un desconocimiento total de lo que sucede al respecto en el resto del continente.

Por consiguiente, tan solo me encuentro en condiciones de ofrecer un informe centrado en su mayor parte en el hispanismo en la Argentina, mientras que lo que he podido reunir de otros países, como Brasil, Chile y Uruguay, se trata sobre todo de datos obtenidos por conocimientos y relaciones personales que deben, por lo tanto, ser tenidos en cuenta como una muestra de las ya señaladas dificultades de integración de los diversos hispanismos que conviven en este rincón austral.

Otro deslinde necesario y por cierto revelador de conflictos subyacentes en las relaciones entre España y los países iberoamericanos, se refiere al concepto de hispanismo, por cuanto definir el objeto de conocimiento con el que voy a trabajar es fundamental para entender algunas cuestiones latentes como problemáticas de identidad.

La pregunta sobre qué se entiende por hispanismo no es tan sencilla de responder y se hace necesario pues trazar primero una suerte de itinerario del imaginario que conlleva su significado. El hispanista argentino Dinko Cvitanovic, en un trabajo de reciente aparición titulado «Hispanismo y globalización» señala, a propósito de esta necesaria definición, la evolución semántica con que aparece registrado el vocablo «hispanismo» en el Diccionario de la R.A.E.:

Mientras hasta no hace mucho aparecía definido como la «afición al estudio de la lengua y literatura españolas y de las cosas de España» (1970), en una edición más reciente (1984) está consignado como la «afición al estudio de lenguas, literaturas o culturas hispánicas». La diferencia que se advierte es pequeña, pero no desdeñable: se ha suplantado el término español por el de hispánico y, en lugar de referirse a una literatura (la española) se pluraliza, en alusión más o menos obvia al contexto de la antigua Hispania, término este último que concierne probablemente a los «pueblos que formaron parte de ella y a los que nacieron de estos pueblos en época posterior». (1999: 22)

Esta puntualización no deja de resultar interesante en razón de que la matización que desde un criterio restrictivo pasa a uno más abarcador 
está asentada en una percepción del problema que mucho tiene que ver con la impronta que el hispanismo que se desarrolla en universidades americanas y europeas de otra órbita lingüística, ha impuesto en los últimos años a su objeto de estudio. En efecto, debido a la enorme gravitación alcanzada por la literatura de los países de Hispanoamérica se ha producido un marcado nivel de interés hacia este ámbito por sobre el de los estudios de la literatura peninsular.

Sin embargo, es evidente que si centramos la atención en nuestra perspectiva como acertadamente afirma Emilia de Zuleta «desde Hispanoamérica, el término hispanismo sigue nombrando, preferentemente, tanto la presencia de lo español en obras americanas como el estudio de la lengua, la literatura y la cultura españolas» (1993: 17). En esta línea de orientación voy a abordar a continuación el panorama que intentará reflejar la situación actual de los estudios hispánicos en estas latitudes australes del continente americano.

Es importante recordar que esta delimitación del campo se encuentra signada por los procesos históricos que dieron lugar a la independencia, pues al fragor de las guerras y los conflictos políticos con que se fueron forjando las identidades nacionales se construyeron también sus literaturas que intentaban deliberadamente apartarse de los modelos impuestos por España en busca de una expresión propia y original. Esto determina que, aun en la actualidad cuando las relaciones comunes han superado todo vestigio de enfrentamientos, un estudioso de la literatura argentina no se considere en nuestro ámbito un hispanista y lo mismo sucede en cualquiera de los países del continente en los que el español es la lengua materna.

Por razones comprensibles y tal como he señalado más arriba, voy primero a trazar los lineamientos de los estudios en los países más próximos de los que cuento con cierta información, para luego ofrecer un más detenido y pormenorizado informe del estado actual del hispanismo en la Argentina.

\section{Tendencias actuales de la investigación}

\subsection{En algunos países del mercosur}

En los últimos años, y como resultado de las desfavorables situaciones económicas de la región se ha iniciado un proceso político de integración de un grupo de países del cono sur de este continente. Forman parte del llamado Mercosur: Brasil, Uruguay, Paraguay y Argentina. En cuanto a Chile, se encuentra en la etapa preliminar de incorporación. El 
problema más significativo que acompaña a este tipo de procesos es sin duda que más allá de los discursos oficiales pregonando la integración latinoamericana, más allá de los ambiciosos proyectos económicos y de la competencia y conflictos entre las industrias que surgen a cada momento, poco es lo que en materia cultural se ha avanzado al respecto.

Un caso sintomático lo constituye Brasil, ya que es el único de los países del Mercosur que no pertenece al área lingüística del español. Por consiguiente, un proceso de reafirmación y desarrollo del hispanismo debería procurar afianzar, de un modo más fehaciente, las relaciones y los nexos entre este país y el resto. Al menos, en relación con la investigación y los estudios en las universidades poco o nada parece haberse hecho, aunque es muy difícil llegar a conocer la situación real dada la escasa por no decir ínfima cantidad de información que poseemos, sobre todo en comparación con la enorme dimensión geográfica y el número de habitantes no menos cuantioso.

En este sentido, y como justificativo de mi falta de conocimiento de los estudios hispánicos en Brasil, apelo a las palabras de Antonio. R. Esteves, quien está encargado de suministrar la información para el Boletín de la A.I.H.:

Seguimos disculpándonos por las muchas faltas que debe tener este informe, resultado, principalmente de las dificultades de comunicación existentes en un país de la dimensión de Brasil. Esperamos poder contar, en el futuro, con la colaboración de compañeros que nos ofrezcan informes que permitan completar el cuadro del hispanismo en nuestro país. (4/97: 36)

Una revisión de la bibliografía reunida permite trazar al menos algunas coordenadas de los intereses y líneas de trabajo que parecen prevalecer. Tanto en el caso de las tesis doctorales consignadas, como entre los libros, hay una notoria preponderancia de los estudios sobre la literatura hispanoamericana, seguidos comprensiblemente por los de lengua y a continuación los de historia, mientras que son muy escasos los dedicados a la literatura española.

En cuanto a las actividades de algunos centros de investigación, puedo señalar que por conocimiento personal de algunos colegas brasileños de la Universidade de Sâo Paulo, como Maria Augusta da Costa Vieira y Mario González a quienes encuentro habitualmente en los congresos internacionales de la A.I.H. o de otras asociaciones y que también han asistido a alguno de los realizados en la Argentina, es evidente que se orientan hacia importantes aspectos de la literatura española del Siglo de Oro: Cervantes y también la novela picaresca. 
Los estudios Hispánicos en algunos países de América...

Es interesante destacar al respecto que en esa Universidad se publica la serie Cuadernos de Recienvenido, Publicaçâo do Curso de Pos-Graduaçâo em Lingua Espanhola e Literaturas Espanhola e Hispano-Americana del Departamento de Letras Modernas de la Faculdade de Filosofia, Letras e Ciências, cuyo editor es Jorge Schwartz, en la que se recogen trabajos de los profesores visitantes del Curso de carácter heterógeneo, según se los define en la nota editorial. Entre los doce números que poseo (1/1966-12/1999) se encuentran trabajos de literatura española de Mario González (2/1966), Edwin Williamson (3/1997) y Edward C. Riley (8/1998). El resto contienen en su mayor parte estudios de temas hispanoamericanos entre los cuales los tres últimos están dedicados a conmemorar el centenario de Jorge Luis Borges (10-12/1999).

También por relaciones personales, pues mantengo contacto con ella, puedo mencionar las investigaciones que la profesora Lygia Rodrigues Vianna Peres, profesora de la Universidade Federal Fluminense, está llevando a cabo acerca de la historia en el teatro del Siglo de Oro y sobre las que ha presentado ponencias en recientes congresos de la especialidad. Con esto debo cerrar lo poco que puedo ofrecer sobre el hispanismo en Brasil, vuelvo a lamentarme a la vez que me disculpo ante tantos colegas que, sin duda, dedicarán sus esfuerzos al estudio y conocimiento de la lengua y la cultura hispánicas e insisto en la necesidad de alcanzar para el futuro una más fluida comunicación sobre los intereses y temas que nos preocupan en común.

Tampoco resulta muy alentador el panorama que puedo ofrecer del Uruguay, pues a pesar de la proximidad resultan poco frecuentes nuestros contactos. Más bien parece que el Río de la Plata, ese río «de sueñera y barro» como decía Borges, se empeña en alejar, distanciar ambas orillas que, aunque apartadas por la naturaleza, han convivido siempre en compartidas historias épicas, políticas, económicas y esencialmente culturales. En el Departamento de Letras Modernas de la Facultad de Humanidades de la Universidad de la República Oriental del Uruguay la profesora Eleonora Basso, que está trabajando sobre el teatro de Federico García Lorca, y el profesor Walter Martínez Bonilla se encuentran a cargo de Literatura española. En cuanto al área de estudios latinoamericanos hay mayores vinculaciones con la Universidad de Buenos Aires y suelen concretarse participaciones en jornadas organizadas conjuntamente. Supongo que las muchas obligaciones, que hacia el final del curso universitario nos sobrepasan, han impedido que, al menos hasta el momento de tener que concluir con este informe, llegara a mis manos el material necesario para aportar datos más concretos.

En cambio la información que he recibido de Chile, gracias a la rápida respuesta del profesor Eduardo Godoy Gallardo, Director del Magis- 
ter en Literatura Hispánica y del Doctorado en Literatura de la Universidad Católica de Valparaíso, me permite al menos ofrecer una perspectiva más puntual del estado actual de los estudios. Es evidente que la oferta de los posgrados, ya sean los más tradicionales doctorados como las más modernas maestrías está canalizando la posibilidad de contar con especializaciones en el área y que, junto a las dos que dirige nuestro gentil colaborador, también se cursan en la Universidad de Chile ambos grados bajo la dirección de la profesora María Eugenia Góngora y en la Universidad de Concepción a cargo del profesor Gilberto Triviños. En la Universidad Austral de Valdivia se cuenta con el Magister en Literatura que dirige el profesor Iván Carrasco y finalmente en la Universidad de Santiago el de Magister en Literatura Hispanoamericana a cargo de Sergio Pereira. Si bien carezco de datos que permitan establecer el número de inscriptos y los ya diplomados, así como los contenidos que se imparten no deja de ser una auspiciosa noticia saber que se abre un interesante panorama en el campo de los estudios hispánicos.

En cuanto a las líneas de investigación en las que está trabajando, Godoy Gallardo me hace saber que junto con Haydée Ahumada Peña centran su atención en la generación del 50 en Chile y que, mientras esta última profesora se orienta hacia la narrativa española de las dos últimas décadas, él investiga sobre la novela española de postguerra como puede confirmarse por los libros de estos estudiosos que consigno en la bibliografía que se incluye al final.

Finalmente es oportuno recordar que el hispanismo chileno cuenta con importantes revistas universitarias que constituyen órganos de difusión de los trabajos como es el caso de la Revista chilena de literatura de la Universidad de Chile, Atenea de la de Concepción y Signos de la de Valparaíso de la que es editor el Prof. Godoy Gallardo y en la que hemos colaborado hispanistas argentinos.

De este modo algo incompleto y ciertamente muy restringido en cuanto a lo que debe de ser la realidad de los estudios hispánicos en algunos países del Mercosur pasaré ahora a trazar el panorama de la disciplina en la Argentina.

\subsection{En la Argentina}

Para situarnos en las coordenadas por las que actualmente se desarrolla el hispanismo, en el momento preciso en que estamos por atravesar los umbrales del próximo milenio, resulta conveniente retrotraer nuestra mirada para plantearnos algunas cuestiones que permitan en- 
Los estudios Hispánicos en algunos países de América...

tender la evolución de este proceso. En un trabajo citado anteriormente, Emilia de Zuleta trazó ya hace algunos años, con la solvencia y rigor académico que la caracterizan, la situación en que se encontraba el hispanismo de Hispanoamérica en el momento de realizarse en Buenos Aires, en 1992, el III ${ }^{\circ}$ Congreso argentino de hispanistas (1993: 17-32).

En esa ocasión, recordaba que la suerte corrida por los estudios hispánicos en nuestro país se asemejaba al Guadiana, «aquel río español que durante largos trechos discurre silencioso en las profundidades de la tierra para luego resurgir a la luz con nuevo vigor fecundante» (1993: 32). Sobre esos procesos cíclicos de ascenso y descenso habría que reflexionar pero no es este el lugar ni la ocasión. En cambio, creo que al concluir 1999, no resulta desatinado proponer una suerte de balance de la evolución experimentada a partir de esa fecha.

Ante todo resulta ya un hecho indiscutible la decidida y afianzada continuidad de la Asociación Argentina de Hispanistas gracias al empeño, la constante preocupación y, por cierto, el enorme trabajo de los integrantes de las Comisiones Directivas que se sucedieron desde 1989. Nuestra asociación reúne a los investigadores y estudiosos del ámbito de la docencia universitaria y también de otros niveles que se dedican a la lengua y la literatura españolas. En un primer encuentro realizado en la Universidad Nacional del Sur en octubre de 1986, se comenzaron los acuerdos preliminares; en las Jornadas en Homenaje a Celina Sabor de Cortazar convocadas por la Universidad Nacional de Salta, en 1987, se suscribió el acta fundacional de la asociación y en el $\mathrm{II}^{\circ}$ Congreso llevado a cabo en Mendoza en la sede de la Universidad Nacional de Cuyo se fijaron las bases jurídicas y organizativas de la institución. Allí se eligieron los miembros de la primera Comisión que presidió por tres años (1989-1992) Emilia de Zuleta. Su gestión fue seguida por la de Dinko Cvitanovic (1992-1995) de Bahía Blanca y Melchora Romanos (19951998) de Buenos Aires, mientras que hasta el año 2001 la presidencia será ejercida por Lila Perrén de Velasco de Córdoba.

De este modo, los encuentros trianuales convocados en distintos centros universitarios del país nos permiten reunirnos en un espacio de estudio y reflexión, que se enriquece con la discusión y es digno de destacar, por las dificultades de toda índole que suponen esas empresas editoriales, que se han publicado las actas de los cinco Congresos por lo que contamos con un importante conjunto de materiales que informan qué y cómo se investiga en nuestro país. Entre los años que van de 1995 a 1998 se comenzó a publicar un boletín anual ( Año 1, 1996; Año 2, 1997; Año 3, 1998) editado por Melchora Romanos Presidente y Gloria Chicote Secretaria en la Facultad de Filosofía y Letras, Universidad de Buenos Aires. 
Una de las consecuencias más valiosas resultantes de esta actividad institucional es que, movidos por el interés que promueven estas reuniones, se han realizado otras de carácter más específico nucleadas, ya sea en torno a aniversarios y homenajes a escritores o, con mayor amplitud, a un período de la literatura española como es el caso de los dos simposios (1991 y 1995) que tuvieron lugar en Mendoza convocados por el Dr. Carlos O. Nállim y del congreso (1997) de Buenos Aires sobre «Letras del Siglo de Oro español».

Por consiguiente, la dinámica de trabajo en el hispanismo argentino se ha visto incrementada de modo muy significativo en estos últimos años y el crecimiento y desarrollo de los estudios que paso a reseñar demuestra que junto a los investigadores ya formados y con experiencia académica y solvencia se van afianzando las nuevas generaciones de estudiosos. En este sentido debe destacarse que los programas de incentivos a los docentes-investigadores, implementados por la Secretaría de Políticas Universitarias del Ministerio de Educación, han contribuido a crear grupos de investigación orgánicos que permiten integrar a los más jóvenes con lo que se logra paliar cierto individualismo reinante en los estudios humanísticos a la vez que establecen controles sobre la marcha de los proyectos y sus resultados.

No intento trazar un exhaustivo mapa de los estudios hispánicos, pues al igual que para los países a los que acabo de referirme sucede también que no me ha sido posible obtener toda la información que hubiera deseado. Las causas han sido muy variadas, por ello es que entiendo que pueda considerarse incompleta la perspectiva que voy a dibujar en este mapa tan extenso que configura la diversidad de nuestros centros de estudio. Esta aclaración previa sirva para disculparme ente mis colegas por las muchas falencias que van a encontrar, ya que, ante la imposibilidad de incluir un pormenorizado detalle de las investigaciones y de las publicaciones en las que vuelcan sus resultados, me he visto forzada a realizar una selección que probablemente parezca insuficiente, en especial, a quienes han sido los responsables de compilarla. He privilegiado fundamentalmente los trabajos específicos sobre hispanismo en el sentido definido al comienzo del presente informe y por lo tanto dejo de lado lo referido exclusivamente a literatura argentina. Para organizar en forma adecuada la exposición voy a seguir el orden alfabético de las ciudades en las que se encuentran las universidades y centros de investigación de referencia.

En Bahía Blanca, en el Departamento de Humanidades de la Universidad Nacional del Sur, las actividades del profesor Dinko Cvitanovic han contribuido en los últimos años a consolidar los estudios hispánicos en nuestro país, pues es uno de los iniciadores del movimiento que culminó 
con la creación y desarrollo de la A.A.H. Desde el Centro de Estudios Hispánicos son muchos los emprendimientos que ha llevado a cabo a lo largo de estos años y una significativa muestra la constituye el Boletín de estudios hispánicos (BOEHI) que publica desde mediados de 1985 y que comenzó siendo una hoja informativa de ocho páginas para alcanzar a partir del $n^{\circ} 20$ (1998) mayor extensión y la incorporación de las secciones de ensayo, ficción y poesía. En cuanto a las investigaciones, su campo de interés se ha orientado últimamente a los estudios del pensamiento, la historia y la cultura argentinas en relación con Europa y en ese línea dirige un equipo interdisciplinario de destacados profesores del PGI (Proyecto Grupo de Investigación) sobre: «La recepción argentina de la problemática europea en el período 1950/1970» del que han aparecido ya dos volúmenes colectivos con contribuciones sobre el tema (1996 y 1999).

En el mismo Departamento de Humanidades, de gran tradición en los estudios sobre literatura medieval española, Graciela Rossaroli de Brevedan, dirige el proyecto: «Didactismo y sátira en la literatura española medieval y en otras literaturas». En relación con este proyecto, ha reunido estudios propios y de otras colaboradoras sobre la proyección de Barlaam e Josafat (1998).

En Buenos Aires, el centro de investigaciones en el que los estudios hispánicos cuentan con más larga historia y fructífera producción es el Instituto de Filología y Literaturas Hispánicas «Dr. Amado Alonso», de la Facultad de Filosofía y Letras de la Universidad de Buenos Aires. Fue fundado en 1923 por acuerdo de Ramón Menéndez Pidal y Ricardo Rojas, y actualmente es dirigido por Ana María Barrenechea, discípula de ese gran filológo recordado en el nombre, que consolidó las bases de la investigación lingüística y literaria aplicada a temas de España y América, y cuyo magisterio se irradió a través de sus discípulos hacia otros países. En este momento, las actividades académicas más importantes están centradas en torno a los proyectos de investigación que cuentan con subsidios de la Secretaría de Ciencia y Técnica de la Universidad de Buenos Aires, en los que participan profesores, jóvenes investigadores y becarios de la Universidad y del Consejo de Investigaciones Científicas y Técnicas (CONICET).

En el área de lengua se encuentran en curso de realización los siguientes proyectos UBACyT:

TF 078: «Las interfaces en la estructura lingüística». Directora: Ofelia Kovacci.

$>$ Fi 004: «¿Desastre, derrame, accidente, incidente? Reformulación textual y léxica en la comunicación sobre medio ambiente». Directora: Guiomar Ciapuscio. 
Fi 011: «Competencia léxica y aprendizaje de términos especializados de las disciplinas académicas por estudiantes universitarios». Directora: Graciela M. Giammatteo.

En el área de literatura española se está trabajando en los siguientes proyectos UBACyT:

TL 08: «De las Ejemplares al Quijote: pensar el realismo cervantino desde la mirada del primer lector». Directora: Alicia Parodi.

$>$ TL 029: «Dramaturgia e ideología de la comedia histórica: de la modernidad hacia el fin del teatro aurisecular». Directora: Melchora Romanos.

$>$ TL 113: «El yo narrativo: espacio y subjetividad en la literatura española de los siglos XIX y XX». Directora: María del Carmen Porrúa.

> TL 62: «La obra de Ramón Gómez de la Serna en la Argentina (1939-1963)». Director: Roberto Yahni.

$>$ TL 038: «Posibilidades de una lectura cultural de la literatura europea de la Edad Media». Directora: María Silvia Delpy (Se incluyen temas de literatura española medieval).

Como puede apreciarse los campos de trabajo son variados y además se inscriben en corrientes críticas que sin abandonar los enfoques tradicionales se ven enriquecidos por los nuevos aportes teóricos. Una significativa muestra de estas posibilidades se puede comprobar en las páginas de Filología que desde que Ana María Barrenechea retomó su dirección, en 1985, ha orientado la publicación de volúmenes monográficos entre los que se encuentran, por ejemplo, el XXVII,1-2 (1994), dedicado a Crítica genética y el XXX,1-2 (1997) a Literaturas comparadas. También se han publicado libros en los que se recogen trabajos de los investigadores como el de reciente aparición del equipo que dirige María del Carmen Porrúa (1999) o el que he coordinado sobre Cervantes con trabajos presentados al Tercer Congreso Nacional «Letras del Siglo de Oro español», celebrado en Buenos Aires en 1997, que tiene como editores responsables a Alicia Parodi y Juan Diego Vila (1999).

Otro de los centros de investigación de la misma Facultad que por su área de interés cuenta con estudiosos que trabajan sobre temas relacionados con el hispanismo es el Instituto de Literatura Hispanoamericana que dirige Noé Jitrik. Entre los proyectos UBACyT vinculados se encuentran:

$>\mathrm{TF}$ 73: «El proceso de automatización de la literatura latinoamericana». Director: Noé Jitrik. 
Los estudios Hispánicos en algunos países de América...

FI 164: «Nudos constitutivos de la modernidad literaria en América Latina, 1880-1916 (Modernismo)». Directora Susana Zanetti.

$>$ JF 12: «La construcción de los espacios americanos: productores y destinatarios (América Meridional en los siglos XVI y XVII)». Directora: Silvia Tieffemberg.

La actividad desarrollada en este Instituto y en el de Filología y Literaturas Hispánicas se enriquece y amplía más allá del marco institucional de la Facultad de Filosofía y Letras por cuanto la participación de los investigadores en congresos nacionales e internacionales, las publicaciones así como la labor docente que realizan, ofrecen una significativa muestra de los alcances del hispanismo en el campo científico y cultural.

Antes de pasar a otro ámbito geográfico, se hace necesario para completar este panorama destacar la meritoria labor que, desde 1978, ha cumplido el Seminario de Edición y Crítica Textual (SECRIT), dependiente del CONICET, del que fue su fundador y director el profesor Germán Orduna. Lamentablemente su reciente fallecimiento (15/12/1999) constituye una pérdida irreparable en nuestro país para la docencia e investigación en el área de la filología española que supo enriquecer con rigurosos y ponderados trabajos y ediciones críticas de textos fundamentales de la literatura española medieval. Su profundo magisterio ha dejado entre sus colaboradores más jóvenes la huella y el fervor necesarios para llevar adelante una tarea para la que hace falta poseer una gran vocación.

Sin duda, quienes colaboraban en el Proyecto de investigación PICT (Agencia Nacional de Promoción Científica y Tecnológica-CONICET) sobre: «La variación lingüística y textual del discurso narrativo en la prosa histórica y ficcional castellana. De la Baja Edad Media al primer Renacimiento», continuarán con su labor ya que tienen la formación necesaria para lograr sus objetivos. Pero, muy especialmente, es de esperar que lleven adelante, con el mismo éxito con que hasta ahora lo han hecho, el proyecto editorial que suponía desde Buenos Aires proyectar a nivel internacional una revista de las características y el alto grado de especialización de Incipit, donde desde 1981 se da cabida a colaboraciones dedicadas a los problemas y métodos de edición y crítica textual de obras españolas y americanas. Estos logros no deben perderse si queremos mantener en un alto nivel de calidad los estudios hispánicos.

En la Universidad Nacional de Córdoba un activo y laborioso grupo de hispanistas que tuvieron a su cargo la organización, en 1998 , del $V^{\circ}$ Congreso Argentino de Hispanistas, liderado por Lila Perrén de Velasco, ha trabajado muy particularmente para esa ocasión en un proyecto sobre: «El ensayo en la generación del 98» cuyos resultados aparecen publicados en las 
Actas de reciente aparición (1999). Es digno de destacar que también son importantes los trabajos que vienen publicando desde hace algunos años sobre poesía española contemporánea. En el área de lingüística, la profesora María Teresa Toniolo dirige un proyecto sobre: «El español hablado en Córdoba - Argentina - a fines del siglo XX. Corpus sociodialectal».

En la Universidad Nacional de La Plata han cobrado nuevo impulso las actividades del área de literatura española, particularmente por la dedicación y capacidad de trabajo de la catedrática Gloria Chicote. Dirige un proyecto trianual sobre: «Alteridad y representaciones culturales en la narrativa española: continuidades y rupturas entre los orígenes y las manifestaciones contemporáneas», en el que se integran investigadores de las dos cátedras de literatura española. Ya se encuentra en prensa el primer número de la revista especializada Olivar, creada para difundir las actividades en el campo del hispanismo nacional e internacional. Se han realizado reuniones académicas con especialistas y dictado cursos de posgrado a cargo de profesores visitantes de España, Alemania y Estados Unidos. Es muy auspicioso comprobar cómo se está desarrollando una tarea que augura un renovado espacio de docencia e investigación.

De igual modo, quiero poner de relieve la importancia que ha cobrado también en estos últimos años el estudio de la literatura española en la Universidad Nacional de Mar del Plata, pues en el Centro de Letras Hispanoamericanas (CELEHIS) de la Facultad de Humanidades se han concretado trabajos de gran significación sobre la poesía española contemporánea por parte del Grupo de investigación de «Semiótica del discurso» que dirige Laura Scarano. Los estudios realizados por el equipo de investigadores que lo integran, desde 1992 hasta 1999, se han proyectado en libros colectivos (1994; 1996 y 1998) y ahondado en tesis doctorales y de maestría. El próximo proyecto que se pondrá en marcha a partir del año 2000 tendrá como objeto de investigación: «Literatura y enunciación. Ficciones y posiciones de sujeto en la literatura española contemporánea».

También como equipo radicado en el CELEHIS, el Grupo «Literatura del Siglo de Oro» que dirige Marta Villarino, ha concretado enfoques originales sobre la comedia de Lope de Vega al centrar su atención en los procedimientos de lectura-escritura-reescritura y la construcción de las representaciones sociales. Los trabajos presentados en Congresos nacionales e internacionales muestran una perspectiva de indagación certeramente orientada hacia algunos de los problemas que son más atendidos hoy por la crítica de la comedia aurisecular. Se propone continuar en esta línea con un proyecto sobre: «El último período de la comedia de Lope de Vega y los dos dramaturgos sobre los que se proyecta: Tirso de Molina y el primer Calderón de la Barca». 
Estos grupos junto con los de «Historia y ficción», dirigido por Elisa Calabrese, y «Latinoamérica: literatura y sociedad», dirigido por Mónica Scarano, consolidan en el CELEHIS una confluencia de estudios sobre el hispanismo, entendido en sentido amplio, que afianzará de modo sólido el área.

En Mendoza, consagrado centro del hispanismo argentino, los investigadores de la Facultad de Filosofía y Letras de la Universidad Nacional de Cuyo, tienen en Emilia de Zuleta una figura de prestigio internacional que desde ese enclave provincial y con auténtica responsabilidad ha sabido convocar encuentros, congresos y homenajes con la intención de afianzar y desarrollar nuevas perspectivas críticas para enriquecer la tradición de los estudios hispánicos en nuestro país. Como Coordinadora general del «Grupo de Estudios sobre la Crítica Literaria» (GEC) ha desarrollado en los últimos diez años, con la colaboración de las investigadoras que la acompañan en esta empresa, una tarea que ella misma ha reseñado en la Presentación del número 9-10 del Boletín del G.E.C. (1998: 9-11) de la que rescato este fragmento para dejar constancia de la importancia de lo realizado

El GEC organizó talleres sobre la traducción, homenajes a Pedro Salinas y Jorge Guillén, un Simposio sobre Mujer, historia y cultura, unas Jornadas Del 98 al 98 y, sobre todo, tres Encuentros sobre teorías y prácticas críticas, realizados en 1991, 1994 y 1997. Publicó las Actas del primero de estos Encuentros y varios libros: entre ellos los que contienen los trabajos leídos durante los Homenajes a Salinas y Guillén y en el Simposio sobre la mujer.

A través de todas estas tareas creemos que se ha ido consolidando una tradición. Una tradición fundada en nuestra actitud frente al trabajo crítico: equilibrio entre el pasado y el presente, apertura científica, pluralismo, comprensión y vocación de diálogo. (1998: 10)

A esto debe añadirse ahora la creación de la serie «Los Libros del GEC» que encabeza el volumen Literatura y conocimiento editado por Mariana Genoud de Fourcade (1998), publicación que recoge los trabajos del proyecto dirigido por esta profesora sobre el tema: «La literatura como modo de conocimiento». En el segundo, Recuerdo y homenaje a Federico García Lorca en su centenario 1898-1998, editado por Gladys Granata de Egües (1999), se han reunido los trabajos del Simposio realizado en Mendoza en junio de 1998. Todo esto demuestra que existe la posibilidad de construir, desde la investigación y la docencia, con el ejercicio de una crítica generadora nacida de la esencia de los estudios hispánicos, nuevas y mayores dimensiones para su integración y ampliación de perspectivas. 
Para ir ya concluyendo, me resta recordar la tarea que en la Universidad de Salta está realizando, en el campo de la literatura española, Graciela Balestrino de Adamo que ha dirigido el proyecto: «Metatexto teórico en la dramaturgia española contemporánea (Buero Vallejo, A. Sastre, Sanchis Sinisterra)». Los resultados de la investigación se presentan en un sugerente libro, escrito en colaboración con Marcela Beatriz Sosa (1997).

Finalmente, en la Universidad Nacional de Tucumán, el Instituto de Literatura española (ILE), dirigido por Aída Francisca Frías de Zabaleta, es el ámbito en el que se desarrolla el Programa de Investigación (26 H/130), aprobado por la Secretaría de Ciencia y Técnica de la UNT, sobre: «Las raíces hispánicas en la cultura tradicional de Tucumán: identidad y mundialización» (1997-2000). Está integrado por cuatro proyectos: 1) Los textos orales en Tucumán: tradición e identidad; 2) El sefardismo. Raíces, tradición e identidad cultural; 3) Estudio de la mujer en la comunidad sirio libanesa de Tucumán: tradición e identidad; 4) Hombre andino: patrimonio e identidad. De estos estudios de orientación cultural se han publicado informes de investigación y documentos de trabajo a través de distintos órganos de difusión del ILE.

Este cuadro de situación de las tendencias de los estudios hispánicos en la Argentina, aunque incompleto ya que ha sido necesario dejar de lado mucho material, ofrece al menos un muestreo suficiente para obtener una visión de conjunto acerca de la importancia y variedad con la que se comienzan a configurar las líneas futuras de trabajo.

\section{Perspectivas de trabajo}

En primer lugar, me parece fundamental insistir en que para integrar el hispanismo en América del Sur, es necesario encontrar formas fluidas de interacción que faciliten de algún modo la relaciones entre los diversos hispanismos nacionales. Como ya he señalado, hay un desconocimiento total de lo que se está haciendo en cada uno de los países y no estaría de más procurar una integración que, de algún modo, funcionara como correlato de lo que se intenta a nivel político y económico en el Mercosur. Esto debe de ser instrumentado a nivel institucional y no solamente por conocimientos personales de los estudiosos. Tal vez, le corresponda a España jugar un papel de importancia en la coordinación de los canales de comunicación necesarios.

En cuanto al pronóstico del desarrollo futuro del hispanismo en nuestro medio, hay que considerar que aunque en los planes de estudio de 
Los estudios Hispánicos en algunos países de América...

nuestras universidades y en la enseñanza media se reduce el lugar asignado a la literatura española, hecho que en buena medida llevó a la creación de la $\mathrm{AAH}$ con el fin de fortalecer los vínculos entre quienes nos dedicamos a su estudio, tal parece que el arrinconamiento ha acrecentado la voluntad de trabajo de los investigadores. Por otra parte, es motivo más que esperanzador la presencia de nuevos y jóvenes estudiosos de primera línea que demuestran ya haber alcanzado una madurez y capacidad prometedora de un destacado papel en el hispanismo internacional.

La continuidad de las publicaciones periódicas de larga o de reciente aparición, junto a la puesta en marcha de las nuevas, permitirá mantener los medios para difundir las investigaciones y crear canales de debate. También resulta auspicioso comprobar la creciente publicación de libros que dan cuenta de los resultados de los proyectos de investigación en los que participan grupos de estudiosos. En este sentido, los subsidios concedidos por las Secretarías de Ciencia y Técnica han permitido concretar publicaciones que los menguados presupuestos universitarios hacían imposible de lograr hace unos pocos años y por el mismo medio se está facilitando la compra de material bibliográfico y de publicaciones periódicas. Es notoria, de igual modo, la posibilidad de contar con ayudas económicas para facilitar viajes que permitan asistir a congresos o realizar investigaciones en el exterior, y la obtención de becas de las universidades o del CONICET para formación, perfeccionamiento y doctorales que han favorecido a interesados en estudios hispanísticos.

En lo que hace a la formación de especialistas en el área de los estudios hispánicos, es de fundamental importancia la creación de posgrados que colaboren en su perfeccionamiento académico. Esto se está alcanzando ya en la Maestría en Letras Hispánicas de la Universidad Nacional de Mar del Plata que dirige Elisa Calabrese, en la que ya comienzan a diplomarse los primeros graduados, algunos de ellos con tesis dedicadas a autores españoles.

En la Universidad de Buenos Aires acaba de aprobarse recientemente la creación de la Maestría en Literaturas Española y Latinoamericana que cuenta ya con la dirección de Noé Jitrik e iniciará sus actividades en el segundo semestre del año 2000. Como objetivo principal se propone crear un ámbito para la investigación de problemas vinculados con las literaturas española y latinoamericana, con especial énfasis en sus interacciones, promoviendo el desarrollo de la capacidad analítica, crítica y creativa de aquellos que se interesen en completar su formación de grado.

También en el posgrado hay que destacar la creciente importancia de doctorandos que se orientan hacia temas de hispanismo y que comienzan 
ya a defender sus tesis que constituyen aportes originales y cuentan con solventes planteos que se concretarán, con seguridad, en libros.

En verdad, hace apenas unos años este panorama resultaba más sombrío y difícil e incierto el pronóstico, pero hoy ante las posibilidades con que se cuenta, limitadas en muchos casos y suficientes en otros, es factible afirmar que existe de cara al futuro más que una esperanza de recuperación del hispanismo en la Argentina.

Porque de futuro se ha de tratar, como ya lo he dicho en otra ocasión, cuando acabamos un siglo y comenzamos un nuevo milenio, quiero recordar estas palabras de Gracián en $E l$ discreto: «Estamos ya a los fines de los siglos. Allá en la Edad de Oro se inventaba, añadióse después; ya todo es repetir. Vense adelantadas todas las cosas, de modo que ya no queda qué hacer, sino elegir». En efecto, elegir el camino que permita continuar con la recuperación de un hispanismo que afianzado en la difícil tradición de ricos antecedentes y destacados maestros se renueve para proyectarse en las nuevas generaciones hacia el porvenir.

\section{Bibliografía}

Ahumada Peá, Haydée. 1999. Poder y género en la narrativa de Rosa Montero. Madrid: Pliegos.

Asociación Internacional de Hispanistas. 1997. Boletín, ${ }^{\circ} 4$.

Asociación Internacional de Hispanistas. 1998. Directorio de socios. Lebanon, New Hampshire: Whitman Communications.

Balestrino de Adamo, Graciela y Marcela B. Sosa, eds. 1997. El bisel del espejo. La reescritura en el teatro contemporáneo español e hispanoamericano. Salta: CIUNSa/ CeSICA.

Cvitanovic, Dinko; Nilsa M. Alzola; Nidia Burgos; Antonio F. Mingorance; Sandro Abate y AdRIANA Ressia, editores. 1996. La Argentina y Europa (1930-1950). Bahía Blanca: Departamento de Humanidades, Universidad Nacional del Sur.

Cvitanovic, Dinko; Sandro Abate; Nidia Burgos; Nilsa M. Alzola; S. Martos; R. Iriarte y Antonio F. Mingorance, eds. 1999. La Argentina y Europa (1930-1950). Bahía Blanca: EDIUNSA. Volumen II.

Cvitanovic, Dinko. 1999. “Hispanismo y GLOBAlización». En Brizuela, Mabel; Cristina Estofán; Gladys Gatti; Silvina Perrero, coords. El hispanismo al final del milenio. $V$ Congreso Argentino de Hispanistas. Asociación Argentina de Hispanistas. Córdoba: Comunicarte. Vol. I, 21-30.

GENOUd DE FourCADE, MARIANA, ed. 1999. Literatura y conocimiento. Estudios teórico-críticos sobre narrativa, lírica y teatro. Mendoza: Facultad de Filosofía y Letras, Univiersidad Nacional de Cuyo.

Godoy Gallardo, Eduardo. 1994. Hora actual de la novela hispánica. Valparaíso: Ediciones universitarias.

GonZÁlez, MARIO. 1996. «Celestina: o diálogo paradoxal». En Cuadernos de Recienvenido, 2. Sâo Paulo: Humanitas/FFLCH/USP. 


\section{Los estudios Hispánicos en algunos países de América...}

GRANATA DE EgüEs, GLAdys, ed. 1999. Recuerdo y homenaje a Federico García Lorca en su centenario. 1898-1998. Mendoza: Municipalidad de Mendoza, Editorial Fundar.

Porrúa, María del Carmen. 1999. Lugares. Estudios sobre el espacio literario. Buenos Aires: Facultad de Filosofía y Letras, Universidad de Buenos Aires.

RILey, Edward C. 1998. "La singularidad de la fama de don Quijote». En Cuadernos de Recienvenido, 8. Sâo Paulo: Humanitas/FFLCH/USP.

Romanos, Melchora, coord.; Alicia Parodi y Juan Diego Vila, eds. 1999. Para leer a Cervantes. Estudios de literatura española Siglo de Oro. Vol. I. Buenos Aires: EUDEBA.

Rossaroli de Brevedan, Graciela, ed. 1998. Pervivencia de Barlaam e Josafat en la literatura hispánica. Bahía Blanca: EDIUNS.

Scarano, Laura; Marcela Romano y Marta Ferrari, eds. 1994. La voz diseminada. Hacia una teoría del sujeto en la poesía española. Buenos Aires: Biblos.

Scarano, Laura; Marcela Romano; Marta Ferrari y Marta Ferreyra, eds. 1996. Marcar la piel del agua. La autorreferencia en la poesía españoal contemporánea. Rosario: Beatriz Viterbo.

Williamson, EDwin. 1997. «La trascendencia de la parodia en el Quijote». En Cuadernos de Recienvenido, 3. Sâo Paulo: Humanitas/FFLCH/USP.

Zuleta, Emilia DE. 1993. "Pasado y presente del hispanismo en Hispanoamérica». En Martínez Cuitiño, Luis y Élida Lois, eds., Actas del III Congreso Argentino de Hispanistas. España en América y América en España. Buenos Aires, Instituto de Filología y Literaturas Hispánicas "Dr.Amado Alonso", Facultad de Filosofía y Letras, UBA. Vol. I, 17-32.

Zuleta, Emilia DE. «Presentación». 1998. En Boletín G.E-C. Números 9-10, 9-11. 